HUB-EP-96/60

November 25,1996

\title{
The Tau-W Coupling
}

\author{
Thomas Hebbeker
}

Institut für Physik der Humboldt-Universitāt zu Berlin, Invalidenstr. 110, D-10115 Berlin, Germany

\author{
Wolfgang Lohmann \\ DESY - Institut für Hochenergiephysik, \\ Platanenallee 6, D-15738 Zeuthen, Germany
}

\section{Abstract}

We interpret the existing experimental knowledge on $\tau$ decays in terms of the vector and axial vector couplings to the $\mathrm{W}$ boson, $V_{\tau}$ and $A_{\tau}$. We deduce $V_{\tau}=1.00 \pm 0.09$ and $A_{\tau}=1.00 \pm 0.09$ in agreement with the Standard Model predictions of 1 . 


\section{Introduction}

Purely leptonic $\tau$ decays provide a clean laboratory for a study of the Lorentz structure of weak charged currents. They can be described in the most general case by ten complex coupling constants [1].

Recent new measurements of the Michel parameters in $\tau$ decays have been published by ALEPH [2], ARGUS [3] and L3 [4]. While these measurements alone are not sufficient to determine the ten complex coupling constants [5], it is instructive to interpret them in terms of a specific model with less free parameters. In particular, we assume the exchange of one charged gauge boson with spin 1 . The $\tau$ decays are then described by vector and axial vector couplings only, which are assumed to be real and are left as free parameters in this analysis.

The fermion currents are of the usual form

$$
\bar{f}^{\prime} \gamma^{\mu}\left(V_{f}-A_{f} \gamma^{5}\right) f
$$

implying helicity conservation. $f^{\prime}$ denotes the $S U(2)$ partner of $f$, for example $f=\tau, f^{\prime}=\nu_{\tau}$. We want to express our results also in terms of the equivalent set of right and left handed charged current coupling constants

$$
L_{f}=\frac{1}{2}\left(V_{f}+A_{f}\right) \quad R_{f}=\frac{1}{2}\left(V_{f}-A_{f}\right)
$$

In the Standard Model those couplings are the same for all leptons and quarks:

$$
V_{f}=1 \quad A_{f}=1 \quad L_{f}=1 \quad R_{f}=0
$$

\section{Formalism}

The matrix element of the decay

$$
\tau^{-} \rightarrow \mathrm{e}^{-} \nu_{\tau} \bar{\nu}_{e}
$$

contains the four couplings

$$
\begin{aligned}
g_{L L} & =\frac{1}{4}\left(V_{e}+A_{e}\right)\left(V_{\tau}+A_{\tau}\right)=L_{e} L_{\tau} \\
g_{R R} & =\frac{1}{4}\left(V_{e}-A_{e}\right)\left(V_{\tau}-A_{\tau}\right)=R_{e} R_{\tau} \\
g_{L R} & =\frac{1}{4}\left(V_{e}+A_{e}\right)\left(V_{\tau}-A_{\tau}\right)=L_{e} R_{\tau} \\
g_{R L} & =\frac{1}{4}\left(V_{e}-A_{e}\right)\left(V_{\tau}+A_{\tau}\right)=R_{e} L_{\tau}
\end{aligned}
$$

as defined in ref. $[1,6]$. The four Michel parameters $\rho, \eta, \xi$ and $\delta^{\prime}=\delta \cdot \xi$ measurable in leptonic $\tau$ decays can be expressed as bilinear combinations of those constants $g_{X Y}$. In our special model we get [7]

$$
\begin{gathered}
\rho=\frac{3}{8}+\frac{1}{N} \frac{3}{8} V_{e} A_{e} V_{\tau} A_{\tau} \\
\eta \equiv 0
\end{gathered}
$$




$$
\begin{aligned}
\xi & =\frac{1}{N}\left(V_{e} A_{e}\left(V_{\tau}^{2}+A_{\tau}^{2}\right)-\frac{1}{2}\left(V_{e}^{2}+A_{e}^{2}\right) V_{\tau} A_{\tau}\right) \\
\delta^{\prime} & =\frac{1}{N} \frac{3}{16}\left(V_{e} A_{e}\left(V_{\tau}^{2}+A_{\tau}^{2}\right)+\left(V_{e}^{2}+A_{e}^{2}\right) V_{\tau} A_{\tau}\right)
\end{aligned}
$$

The normalization constant appearing in these formulae is

$$
N=\frac{1}{4}\left(V_{e}^{2}+A_{e}^{2}\right)\left(V_{\tau}^{2}+A_{\tau}^{2}\right)
$$

It equals one in the Standard Model. The parameter $\eta$ is zero in our model and therefore not useful here. The electronic partial decay width $\Gamma_{e}$ is proportional to the normalization constant $N$ :

$$
\Gamma_{e}=\frac{m_{\tau}^{5} G_{F}^{2}}{192 \pi^{3}} \cdot N \cdot(1+\Delta)
$$

The term $\Delta$ contains mass and electroweak radiative corrections $[6,8]$. Including these and using $m_{\tau}=\left(1777.00_{-0.27}^{+0.30}\right) \mathrm{MeV}[7]$ the partial wid th becomes

$$
\Gamma_{e}=4.033 \cdot 10^{-4} \mathrm{eV} \cdot N
$$

The relative uncertainty is smaller than $0.1 \%$ and therefore negligible.

For the leptonic decays into muons analogous relations hold. The higher mass reduces the muonic partial width slightly with respect to the electronic partial width:

$$
\Gamma_{\mu}=3.922 \cdot 10^{-4} \mathrm{eV} \cdot N
$$

From hadronic $\tau$ decays such as

$$
\tau^{-} \rightarrow \pi^{-} \nu_{\tau}
$$

the chirality parameter $\xi_{h}$ has been measured, which can be interpreted as the helicity of the (massless) neutrino:

$$
\xi_{h}=-2 \frac{V_{\tau} A_{\tau}}{V_{\tau}^{2}+A_{\tau}^{2}}
$$

Therefore we have enough measurements to determine $V_{\tau}$ and $A_{\tau}$.

We define here $\operatorname{sign}\left(A_{f}\right)=+1$, since only the relative sign between $V$ and $A$ is meaning ful.

\section{Data}

From the measured lifetime and leptonic branching fractions [7] we obtain

$$
\Gamma_{e}=\frac{B_{e}}{\tau_{\tau}}=(4.03 \pm 0.03) \cdot 10^{-4} \mathrm{eV} \quad \Gamma_{\mu}=\frac{B_{\mu}}{\tau_{\tau}}=(3.92 \pm 0.03) \cdot 10^{-4} \mathrm{eV}
$$

The measurements of branching fractions and of the lifetime contribute about the same fraction to the errors. Note that the errors of $\Gamma_{e}=B_{e} / \tau_{\tau}$ and $\Gamma_{\mu}$ are correlated due to the common factor $\tau$ lifetime $\tau_{\tau}$. 


\begin{tabular}{c|cccc} 
& $\rho$ & $\xi$ & $\delta^{\prime}$ & $\xi_{h}$ \\
\hline$\rho$ & 1 & -0.05 & -0.06 & -0.16 \\
$\xi$ & & 1 & 0.06 & 0.15 \\
$\delta^{\prime}$ & & 1 & 0.26 \\
$\xi_{h}$ & & & & 1
\end{tabular}

Table 1: Correlation coefficients.

A) Assuming $e-\mu$ universality the relevant Michel parameters and the chirality parameter have been measured by ALEPH [2], ARGUS [3] and L3 [4]. Older measurements of $\rho$ have substantially larger errors [7] and are therefore not taken into account. From a fit to the published numbers and correlation matrices we determine the averages:

$$
\rho=0.761 \pm 0.023 \quad \xi=1.055 \pm 0.092 \quad \delta^{\prime}=0.788 \pm 0.071 \quad \xi_{h}=-1.003 \pm 0.022
$$

and the correlation coefficients as shown in table 1. The measurements of $\Gamma$ are independent of the Michel parameter and neutrino helicity determinations.

B) Without any assumption on lepton universality one has to consider the following independent parameters:

$$
\rho_{e} \quad \xi_{e} \quad \delta_{e}^{\prime} \quad \rho_{\mu} \quad \xi_{\mu} \quad \delta_{\mu}^{\prime}
$$

in addition to the partial widths and the neutrino helicity. Those Michel parameters have been measured, too [2-4], but the correlation coefficients are not published. Therefore we will not explore this scenario.

\section{Analysis and Results}

We make the assumption that $V_{e}=A_{e}=V_{\mu}=A_{\mu}=1$ which is consistent with the analyses of $\mu$-decay, $\pi$-decay and neutrino interactions $[1,7]$. This allows us to use measurements of both leptonic decay modes. The formulas simplify:

$$
\begin{gathered}
N=\frac{1}{2}\left(V_{\tau}^{2}+A_{\tau}^{2}\right) \\
\rho=\frac{3}{8}+\frac{3}{8} \cdot 2 \frac{V_{\tau} A_{\tau}}{V_{\tau}^{2}+A_{\tau}^{2}} \\
\xi=2-2 \frac{V_{\tau} A_{\tau}}{V_{\tau}^{2}+A_{\tau}^{2}} \\
\delta^{\prime}=\frac{3}{8}+\frac{3}{8} \cdot 2 \frac{V_{\tau} A_{\tau}}{V_{\tau}^{2}+A_{\tau}^{2}}
\end{gathered}
$$

The Michel parameters $\rho, \xi$ and $\delta^{\prime}$ as well as $\xi_{h}$ all measure the same quantity

$$
2 \frac{V_{\tau} A_{\tau}}{V_{\tau}^{2}+A_{\tau}^{2}}=2 \frac{V_{\tau} / A_{\tau}}{1+\left(V_{\tau} / A_{\tau}\right)^{2}}=\frac{L_{\tau}^{2}-R_{\tau}^{2}}{L_{\tau}^{2}+R_{\tau}^{2}}
$$


i.e. the ratio $V_{\tau} / A_{\tau}$.

From a complete analysis in form of a $\chi^{2}$-fit to all data including the partial widths and taking into account the correlations we obtain the $68 \%$ contours displayed in fig. 1 and

$$
V_{\tau}=1.00 \pm 0.09 \quad \text { or } \quad A_{\tau}=1.00 \pm 0.09
$$

The fit is good with $\chi^{2} / d o f=0.3 / 2$. The correlation coefficient between $V_{\tau}$ and $A_{\tau}$ is equal to 0.999. Equivalently:

$$
L_{\tau}=0.999 \pm 0.003 \quad \text { or } \quad R_{\tau}=0.00 \pm 0.08
$$

with a correlation coefficient of 0.002 . This result is in excellent agreement with the SM expectations and confirms lepton universality. The following upper limit on the contribution from right handed currents can be set:

$$
\left|R_{\tau}\right|<0.13(90 \% \mathrm{CL})
$$
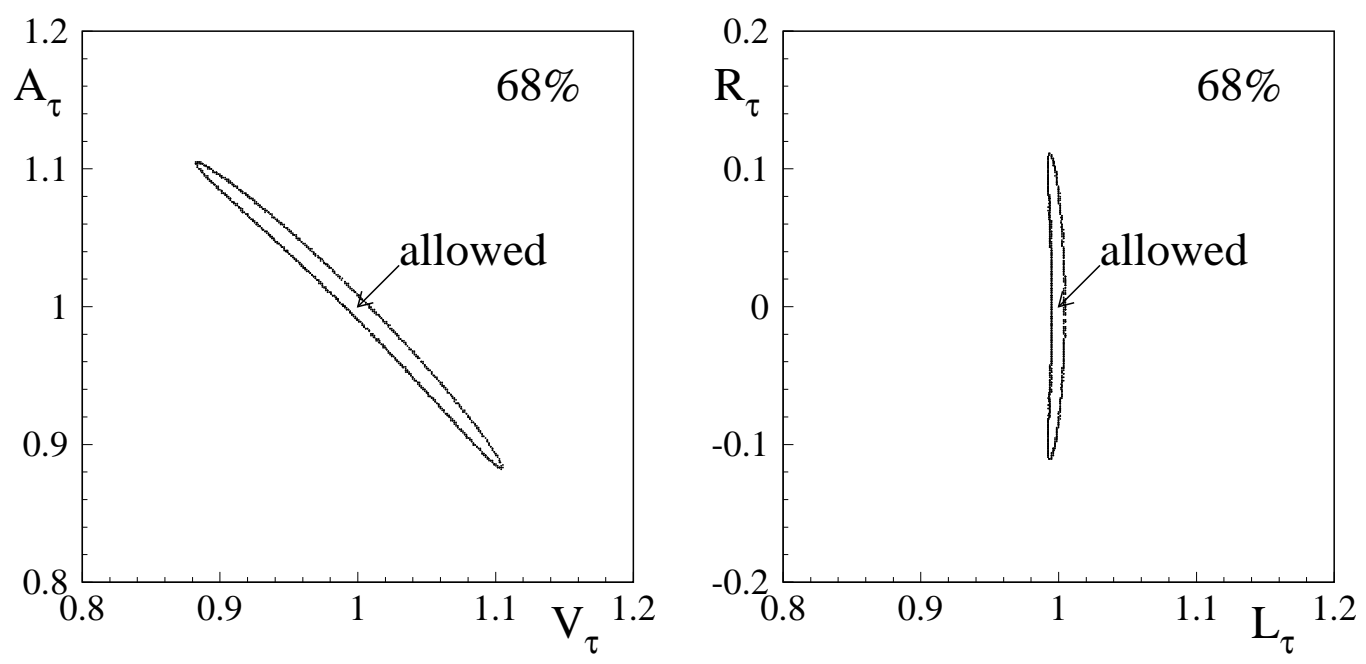

Figure 1:

Figure 2 reveals which constraints on the $W-\tau$ couplings can be obtained using only one or two out of the 6 input parameters (and without imposing a sign convention yet). Fig. 2 a) shows the circular contour determined from the partial widths alone. Fig. 2 b) shows the constraint given by the measurement of $\xi_{h}$. The other parameters $\rho, \xi$ and $\delta^{\prime}$ lead to similar (but wider) contours.

\section{Summary and Conclusions}

Assuming the charged current Lorentz structure to be of a pure (axial)vector nature we have determined the couplings $V_{\tau}$ and $A_{\tau}$ from recently measured $\tau$ parameters.

The errors are now smaller than 0.1 . This is a major improvement with respect to the situation a few years ago. However, the charged current couplings of the $\tau$ are much less well known than the neutral current $\tau$ couplings to the $Z$ [9], where a precision of about 0.01 has been reached [7]. 

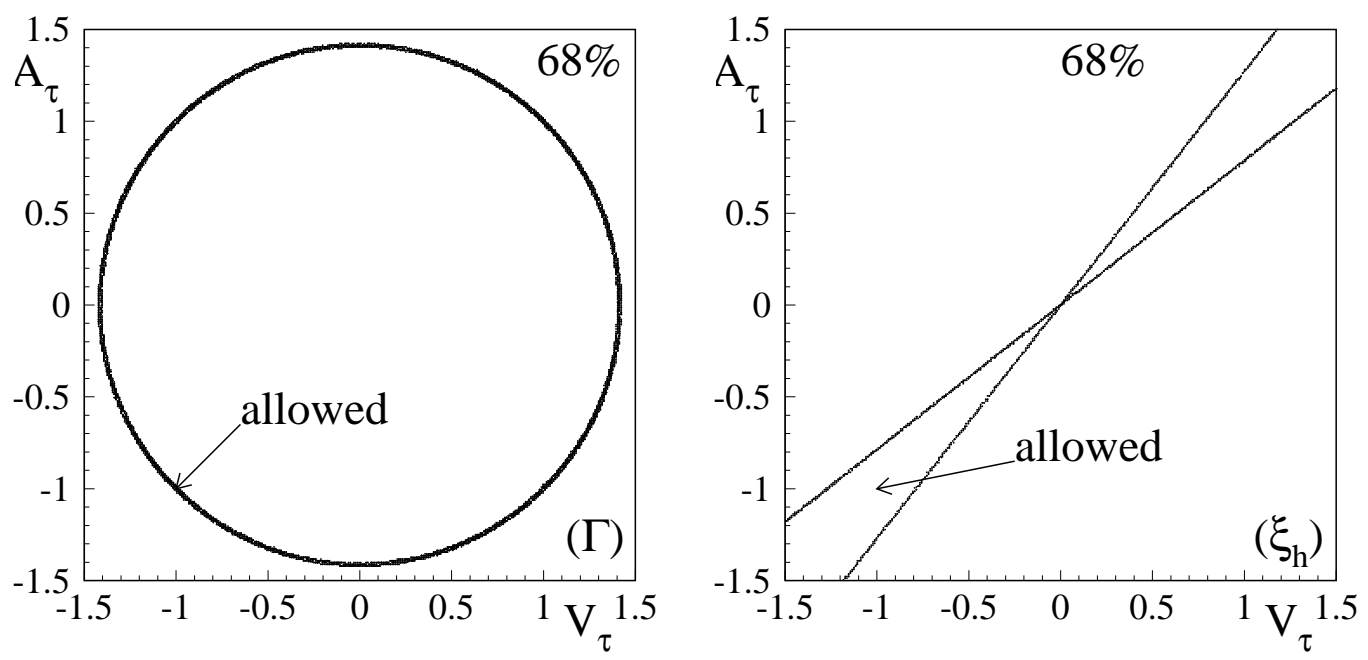

Figure 2:

Small improvements on the charged current couplings will come in the near future from the ongoing analyses of LEP data. The next jump in precision has to wait for a $\tau$-factory or a b-factory.

\section{Acknowledgements}

We would like to thank Achim Stahl and Tord Riemann for several valuable comments.

\section{References}

[1] W. Fetcher, Phys. Rev. D 42 (1990) 1544.

[2] ALEPH Collaboration, D. Buskulic et al, Phys. Lett. B 346 (1995) 379 and Phys. Lett. B 363 (1995) 265 (Erratum).

[3] ARGUS Collaboration, H. Albrecht et al, Phys. Lett. B 349 (1995) 576.

[4] L3 Collaboration, M. Acciarri et al, Phys. Lett. B 377 (1996) 313.

[5] W. Lohmann and J. Raab, DESY 95-188.

[6] A. Stahl, Phys. Lett. B 324 (1994) 121. Note that the formula for $\rho$ is incorrect.

[7] Particle Data Group, R.M. Barnett et al., Phys. Rev. D 54 (1996) 1, and references therein.

[8] W.J. Marciano and A. Sirlin, Phys. Rev. Lett. 61 (1988) 1815.

[9] The LEP Collaborations and the LEP Electroweak Working Group, P. Antilogus et al., CERN preprint CERN-PPE/95-172. 